\title{
Utility-Based Wireless Routing Algorithm for Massive MIMO Heterogeneous Networks
}

\author{
Wei Zhao ${ }^{1,2}$ and Wen-Hsing Kuo ${ }^{2, * \mathbb{D}}$ \\ 1 College of Electrical Engineering and Automation, Fuzhou University, Fuzhou 350108, China; \\ aczhaowei01231994@gmail.com \\ 2 Department of Electrical Engineering, Yuan Ze University, Taoyuan 320, Taiwan \\ * Correspondence: whkuo@ee.yzu.edu.tw
}

Received: 28 September 2020; Accepted: 14 October 2020; Published: 17 October 2020 updates

\begin{abstract}
With the development of 5G communication, massive multiple input multiple output (MIMO) technology is getting more and more attention. Massive MIMO uses a large amount of simultaneous transmitting and receiving antennas to reduce power consumption and raise the level of transmission quality. Meanwhile, the diversification of user equipment (UE) in the $5 \mathrm{G}$ environment also makes heterogeneous networks (HetNets) more prevalent. HetNets allow UE of different network standards to access small cells, while the base stations of small cells access a macro base station (BS) to form a multihop wireless heterogeneous backhaul network. However, how to effectively combine these two technologies by efficiently allocating the antennas of each BS during the route construction process of heterogeneous wireless backhaul networks is still an important issue that is yet to be solved. In this paper, we propose an algorithm called preallocated sequential routing (PSR). Based on the links' channel conditions and the available antennas and location of BSs, it builds a wireless heterogeneous network backhaul topology and adjusts each link's transmitting and receiving antennas to maximize total utility. Simulation results showed that the proposed algorithm significantly improved the overall utility and the utility of the outer area of heterogeneous networks.
\end{abstract}

Keywords: fifth generation; wireless backhaul routing; massive MIMO; utility

\section{Introduction}

Multiple input multiple output (MIMO) refers to the configuration of multiple antennas at the receiving and transmitting terminals to form a system with multiple channels. It is regarded as a promising key technology for fifth-generation (5G) communication systems [1]. Base on MIMO, massive MIMO configures a large number of antennas (hundreds or even thousands) at the transceiver terminals. Massive MIMO has lots of promising benefits, such as high spectral efficiency in a specified number of base stations (BSs) and high data rate [2]. Heterogeneous network (HetNet) is another advantageous concept for wireless backhaul in 5G due to different user equipment (UE) of different network standards allowing access [1]. However, it also has some technical challenges, for example, the strong interference between cells caused by the full frequency multiplex of a large number of users and the limitations of the bandwidth and delay of the wireless backhaul link [3]. Massive MIMO communication within HetNet wireless backhaul with adjustable network topology and the number of transceiving antennas can effectively reduce signal interference and increase channel capacity.

Typically, base stations in HetNets are connected by a tree-shaped topology because of the following reasons: (1) the explosive growth of traffic requires a large number of access points, so an easy-to-expand topology is needed and a tree topology can easily add new nodes to the network; (2) a multihop tree-shaped network is easier to control because a node is only responsible for those that are directly connected to it (i.e., children nodes). To provide a multihop connection to the 
wired network, the macro base station (MBS) serves as the root of the tree. However, many possible tree-shaped topologies can be considered, and each of them may result in different antenna allocations and respective performance impact. Each non-MBS shares the total data rate to its child nodes. All non-MBSs dedicate their antennas to their own parent links, while some BSs have some child links and thus have to adjust antennas between multiple links. Figure 1 shows an example where seven BSs form a backhaul network. $B S_{1}$ serves as the MBS and is connected to the wired network, while each BS uses its respective radio interface to serve UE within their coverage. The topology of the network can be formed according to the red or green tree, while nodes in each tree need to have different links with different numbers of allocated antennas. To decide an optimal tree that can efficiently utilize radio resources and effectively serve each $\mathrm{BS}$, two key issues have to be considered at the same time: topology decision and antenna allocation. Existing topology decision schemes do no support massive MIMO and thus do not consider antenna allocation. On the other hand, existing antenna allocation schemes are established based on a fixed topology. Moreover, the nonlinear nature of the utility gain of each additional antenna makes this problem more challenging. In other words, doubling the number of antennas does not double the utility gain.

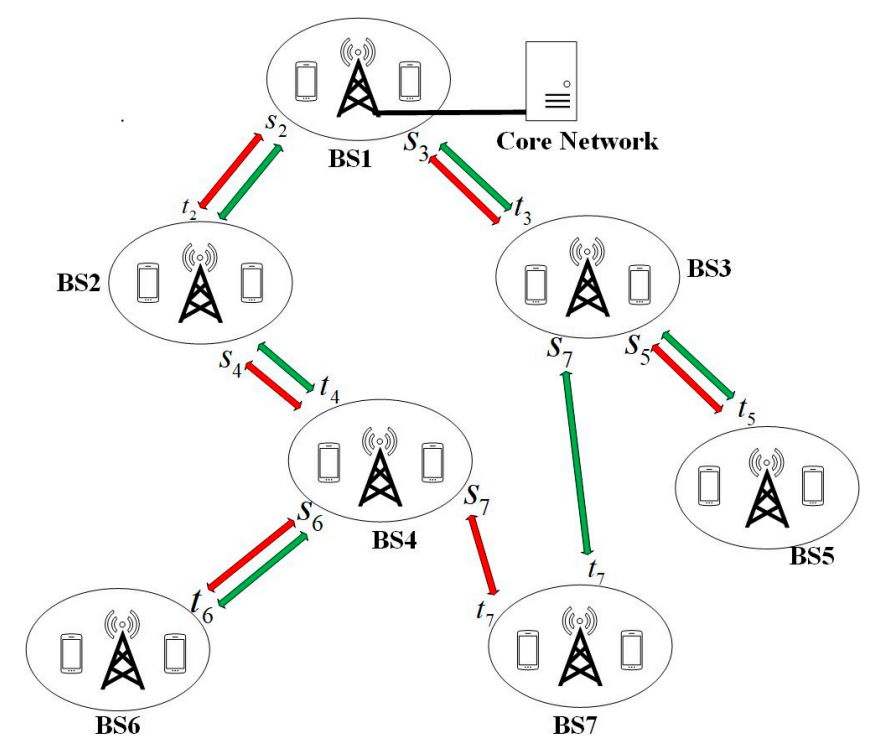

Figure 1. Example of utility-based wireless backhaul routing (UWBR).

Based on the above, taking into consideration antenna allocation while establishing the topology is a challenge. To tackle this problem, we propose a heuristic algorithm named preallocated sequential routing (PSR). PSR adjusts the link of the BSs according to the distance of the root node. Based on the connection state of the previous step, the initial state of the next connection can be determined until all BSs are successfully connected.

The rest of the paper is organized as follows. Section 2 analyzes related work. Section 3 introduces models and formally defines the problem. In Section 4, we propose a heuristic algorithm and analyze its performance. Section 5 presents the simulation results. Finally, we present the conclusion and future work of this study in Section 6.

\section{Related Work}

Several papers have studied the rate, delay, and interference of wireless backhaul transmission. Tabassum et al. [4] analyzed the performance and feasibility of full-duplex (FD) BSs. They proposed a theoretical framework that can reflect different interference problems and accurately analyze a scenario of complex interference full-duplex wireless backhauling. Beyranvand et al. [5] focused on the latency and reliability issues of mobile backhaul networks and proposed a distributed load-balancing 
algorithm for fiber-wireless-enhanced LTE-A HetNets. This method reduced the probability of latency and congestion through distributed load balancing. Chen et al. [6] considered the performance and cost of wireless heterogeneous cellular networks and proposed a superimposed independent homogeneous Poisson point process model for hierarchical networks. In addition, a delay-based access control strategy was proposed to obtain better delay performance. Hong et al. [7] studied the capacity of wireless backhaul networks and proposed a scheme to increase transmission capacity as well as reduce delay. The scheme concatenates small packets into large frames and then use an intelligent channel selection algorithm between adjacent nodes to send large frames.

Many studies have been carried out for spectrum allocation of massive MIMO. Yang et al. [8] optimized the channel performance in situations where the number of available pilot sequences cannot satisfy the demand for users in the massive MIMO system. This issue was modeled as a partially observable Markov decision process. Mosleh et al. [9] studied downlink resource allocation to enhance user experience and improve system performance. In a scenario of cell-free massive MIMO, they proposed an iterative algorithm that can effectively solve the original downlink issue. Zhang et al. [2] investigated the total rate gain in the case of time-division duplex transmission brought by power allocation. Nguyen et al. [10] studied the issue of maximum system energy efficiency in a multiuser and multicell massive MIMO network. They proposed an iterative algorithm to solve the issue of power allocation and optimization of the number of antennas. Moreover, these studies [11-14] also focused on the spectrum allocation of massive MIMO. Khan et al. [15] proposed a novel approach to decrease the overhead of both uplink and downlink channel estimation in massive MIMO systems. Different from traditional algorithms, they used multiple dimensional parameters to reduce the channel estimation overhead. Saraereh et al. [16] focus on channel estimation in massive MIMO- Orthogonal Frequency Division Multiplexing (OFDM) systems. In order to reduce the adverse impact on the channel state information (CSI) due to the pilot overhead being too large, they proposed an algorithm named structured sparse adaptive coding sampling matching pursuit (SSA-CoSaMP) to optimize the channel estimation performance.

Some studies have focused on wireless backhaul topology. Ogawa et al. [17] demonstrated that a multihop network is better than a direct backhaul network in a cellular composite network topology. Furthermore, they proposed an algorithm for backhaul routing, that is, determining the switching status of small cell BSs based on traffic allocation. The algorithm can reduce energy consumption while meeting user traffic demands. Li et al. [18] proposed an algorithm that can improve system efficiency by considering various aspects (i.e., link cross, small angle) of wireless backhaul networks. Moreover, these studies [19-21] also focused on wireless backhaul routing.

To the best of our knowledge, there are no existing studies that focus on the abovementioned issue. In this paper, we introduce the new problem of utility-based wireless backhaul routing (UWBR). Given the number of UE of each BS, the available antennas, and the distance between all BSs, UWBR involves dynamically adjusting the allocation of antennas while selecting routes to improve overall satisfaction of users. To quantify the satisfaction of users, we used a utility function to describe the bandwidth requirements of each BS. In many papers, such as [22-24], utility functions were used to describe the degree of satisfaction of receiving a certain amount of traffic [25].

\section{Utility-Based Wireless Backhaul Routing}

In the section, we formally describe the system model and the notations used in this paper and formulate the problem we mentioned.

\subsection{System Model and Notations}

The system is modeled as follows. We denote with $\mathbf{B S}=\left\{B S_{1}, B S_{2}, B S_{3}, \ldots, B S_{I},\right\}$ the set of BSs, where $I$ is the number of BSs. Each BS has a different number of UE that stays within its coverage and receives its service using conventional radio interface, such as $4 \mathrm{G}$. These user devices mainly require a large amount of downlink traffic for applications such as web-browsing, real-time/non-real-time video 
playing, and location-based information services. $B S_{1}$ represents the MBS that connects to the core network through a wired link and provides other BSs with internet access through wireless massive MIMO antennas. Without losing generality, each BS is indexed in the increasing order of its distance to $B S_{1}$. In other words, for all $i>j, B S_{i}$ is more distant to $B S_{1}$ than $B S_{j}$. Next, $N_{i}$ is the number of antennas of each $B S_{i}$. In order to connect each BS to the backhaul, a tree-shaped topology needs to be established. This topology is described as follows: for each $B S_{i}$, let $\hat{i}$ be the index number of its parent node and $\breve{i}$ represent the set of its child nodes. To avoid inefficient links, the BS's children can only be more distant nodes, that is, for all $j \in \check{i}, j>i$. Next, to uniquely identify each link, each link is indexed with its child-side node. Therefore, link $l_{i}$ indicates the link between $B S_{i}$ and $B S_{\hat{i}}$ and $t_{i}$ is the antennas on the child-side node (i.e., uplink), while $s_{\hat{i}}$ refers to the present-side antennas (i.e., downlink). Based on the tree-shaped topology, an allocation $A_{i}=\left\{t_{i}, s_{j}\right.$ for all $\left.j \in \check{i}\right\}$ indicates each $l_{i}$ 's antenna allocation, while $r_{i}^{\Sigma}$ denotes the total uplink data rate of each $B S_{i}$, which is shared by $B S_{i}$ and all the nodes belonging to its subtree.

Next, the communication channels and data rates are modeled as follows. In general, the channel model of MIMO is represented by a $M \times K$ matrix, where $M$ and $K$ mean the number of transmitting and receiving antennas, respectively. It describes the performance of each subchannel. Nonetheless, considering the complexity of the problem, it is assumed that the antenna gains of the same BS are approximately equal. This approximation is reasonable when the number of antennas is large. Based on [11], Equation (1), the channel model is set as follows:

$$
l_{i}=\min \left(s_{i}, t_{i}\right) \cdot \frac{B}{N_{i}} \cdot \log _{2}(1+\mathrm{SINR}),
$$

where $B$ is bandwidth. As the focus of this paper is on utility and backhaul routing, the channel quality is set to only Gaussian white noise. Basing on the above assumption, SINR (Signal to Interference plus Noise Ratio) is set as follows:

$$
\frac{\frac{P}{N_{i}} \cdot \alpha}{\sigma}
$$

where $P, \alpha$ and $\sigma$ are the emitting power $(\mathrm{W})$, path loss coefficient, and Gaussian white noise, respectively. Referring to a path loss coefficient model in [26], $\alpha=10^{-\left(14+3.67 \log d_{i, j}\right)}$, where $d_{i, j}$ is the distance between $B S_{i}$ and $B S_{j}$ in kilometers. Based on [21], Equation (5), each BS's utility model is set as follows:

$$
U_{i}(r)=f_{i} \cdot \frac{\log (r+1)}{\log (10)}
$$

where $f_{i}$ is the loading factor presenting the number of $B S_{i}$ 's associate $\mathrm{UE}$, and $r$ is the data rate.

Figure 1 illustrates the notations, where a wireless backhaul scenario with an MBS $\left(B S_{1}\right)$ and 6 BSs $\left(B S_{2}-B S_{7}\right)$ is considered. Taking the red topology as an example, the link between $B S_{1}$ and $B S_{2}$ is $l_{2}$. $\check{2}$ presents $\left\{B S_{1}\right\}$ and $\hat{2}=B S_{4}, A_{2}=\left\{t_{2}, s_{4}\right\}$. In a similar way, $l_{6}$ refers to the link between $B S_{4}$ and $B S_{6}$, while the link between $B S_{4}$ and $B S_{7}$ is $l_{7} ; \hat{4}$ presents $B S_{2}$ and $\breve{4}=\left\{B S_{6}, B S_{7}\right\}, A_{4}=\left\{t_{4}, s_{6}, s_{7}\right\}$. On the other hand, if the green topology is decided, $\hat{7}=B S_{3}$ instead of $B S_{3} ; A_{4}$ and $A_{3}$ become $\left\{t_{4}, s_{6}\right\}$ and $\left\{t_{3}, s_{5}, s_{7}\right\}$, respectively.

\subsection{Problem Formulation}

Given the above notations, the UWBR problem involves the following: given

$$
\text { BS, }\left\{N_{i}, U_{i}(\cdot), l_{i}(\cdot, \cdot)\right\} \text { for all } i \text {, }
$$

find

$$
\hat{i}, \breve{i}, A_{i}=\left\{t_{i}, s_{j} \text { for all } j \in \breve{i}\right\} \text {, for all } i \text {, }
$$


to maximize

$$
\sum_{i \in \mathbf{B S}} U_{i}\left(r_{i}\right)
$$

subject to for all $i$,

$$
\begin{gathered}
r_{i}^{\Sigma}=l_{i}\left(s_{i}, t_{i}\right), \\
t_{i}+\sum_{j \in \grave{i}} s_{i} \leq N_{i}, \\
r_{i}=r_{i}^{\Sigma}-\sum_{j \in \bar{i}} r_{j}^{\Sigma} \geq 0, \\
\text { for all } j \in \check{i}, \quad j>i
\end{gathered}
$$

Given the geographical distribution of BS, each $B S_{i}{ }^{\prime}$ s available antennas $N_{i}$, best-effort demand $U_{i}(\cdot)$, as well as its channel condition $l_{i}(\cdot, \cdot)$ is considered. UWBR includes finding the parent node of each BS and assigning antennas on both sides of each link to maximize the traffic of wireless network utility $\sum_{i \in \boldsymbol{B} S} U_{i}\left(r_{i}\right)$. The problem has the following constraints: (7) the maximum link data rate of each BS depends on the transceiver antennas on both sides of the link; (8) the sum of the uplink and downlink antennas of each BS cannot exceed its total antennas; (9) the uplink speed of the BS serving its own UE is the total link data rate minus the total speed of the child nodes and it is non-negative, that is, (9); (10) the child node of each BS must be more distant to the MBS.

UWBR has an NP-complete complexity, as shown in Appendix A.

The entire system's utility of UWBR is bounded by $(I-1) \cdot U\left(\frac{l_{2}\left(N_{i}, N_{i}\right)}{I-1}\right)$, as shown in the proof of Appendix B.

\section{Preallocated Sequential Routing}

Here, we propose a heuristic algorithm called PSR that contains three parts. Algorithm 1 generates an initialized topology and respective antenna allocation, which connects all BSs to MBS directly. In this topology, MBS allocates all antennas to BSs with maximal incremental utility. Then, the main function, shown in Algorithm 2, traverses each BS and compares the utility of possible parent nodes and finally connects the node that has the maximal presumed utility as the parent. Algorithm 3 shows the subfunction, which calculates the presumed utility of the subtree in which the candidate node is located and returns it to the main function for comparison. Figure 2 presents a flow chart diagram of PSR.

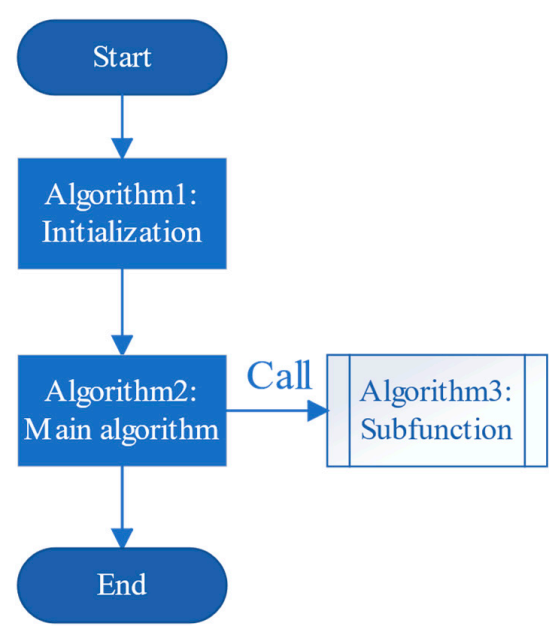

Figure 2. Flow chart diagram of preallocated sequential routing (PSR). 


\section{Proposed Algorithm}

The system constructs an initial antenna allocation in the first place. In order to evaluate the utility of each BS, all non-MBSs are directly connected to the MBS $B S_{1}$. Then, antennas are preallocated to BSs. $D_{i, j}$ is a two-dimensional matrix composed of the distance between two BSs. Each BS will get initial preallocated antennas for subsequent topological connection.

First, the initialization algorithm sets $U_{M A X}$ (maximum system utility) and $U_{\text {total }}$ (utility after $\mathrm{N}$-th antenna allocated) as zero (Step 1-2). Subsequently, in each round of the first for loop (Step 3-22), PSR allocates one antenna to the BS with the maximum utility. To achieve this, in the second for loop (Step 4-20), it calculates the utility of each BS after adding an antenna. Then, PSR assigns one antenna to $B S_{i}$ temporarily and stores the number of antennas in $s_{t}(i)$ (Step5). Then, in the third for loop (Step 6-14), the total utility of the system after accumulating the antenna is calculated. This loop calculates the total energy efficiency, $U_{\text {total }}$, of the system. When the utility of $B S_{i}$ is added, the antenna of $s_{t}$ is used (Step 7-9), and when the utility of $B S_{j}$ is added, $s(i)$ is used (Step 10-12). After $U_{\text {total }}$ is obtained, Step 15 compares $U_{\text {total }}$ with $U_{\text {MAX }}$. If $U_{\text {total }}$ is larger, it becomes the new $U_{\text {MAX }}$ (Step 16), and then $i$ is temporarily stored in $i_{S}$ (Step 16). Then, $U_{\text {total }}$ is set to zero for the next round, while $U_{M A X}$ retains the result as the maximum value of the previous round of the system. After the second for loop is over, the antenna is allocated to the $B S_{i_{s}}$ that can obtain maximum utility (Step 21). The $N_{i}$ antennas are allocated in sequence until the allocation is completed, and the initial preallocated antenna set, $s(i)$, is obtained.

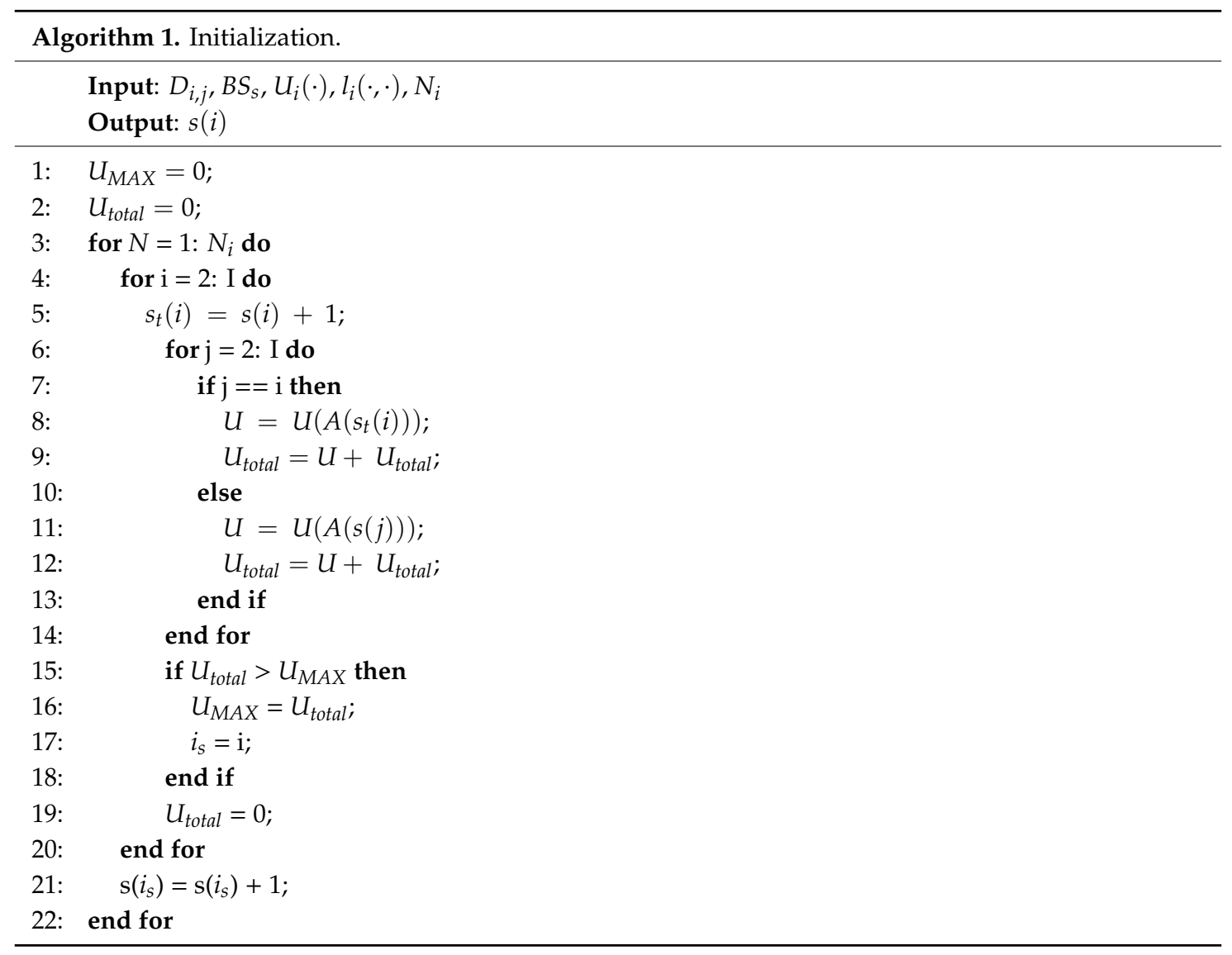

Next, we detail the main function. PSR has to calculate each $B S_{i}$ in the increasing order of its distance to MBS. $S E T_{I}=\left\{B S_{I}, \ldots\right\}$ is the set of the $i$-th BS's subtree, where $B S_{I}$ is a non-MBS root node, and it is then redistributed according to the subfunction.

In the main algorithm, PSR cycles through all BSs to adjust the parent nodes of all BSs in each round of the first for loop (Step 2-16). $\Delta U^{M A X}$, the median value of the subsequent utility, is initialized 
as zero (Step 3). In Step 4, the candidate node set $V_{i}$ of the $B S_{i}$ is listed, which contains all the BSs $B S_{i}$ whose distances to $B S_{i}$ and $B S_{1}$ are not larger than the original $D_{i, 1}$. This is because if $B S_{i}$ is connected to any $B S_{j}$ where $D_{i, j}$ is greater than $D_{i, 1}$, then under the same antenna allocation, $U\left(l_{i, j}(A)\right) \leq U\left(l_{i, 1}(A)\right)$. Therefore, choosing a BS closer to MBS and having $D_{i, j}<D_{i, 0}$ will have a better chance of obtaining a larger utility.

In the second for loop (Step 5-14), PSR needs to analyze the utility of the candidate nodes in $V_{i}$ in turn. If the $B S_{i}$ is connected to the $m$-th candidate node, the antenna allocation of the subtree where the candidate node is located will change accordingly. As all BSs will eventually be connected to the MBS through the multihop network, the first BS that is non-MBS is specified as the subtree root node, that is, $m_{\alpha}$ (Step 6). Next, Step 7 accumulates the preallocated antennas obtained by $B S_{i}$ to $m_{\alpha}$. Then, the subfunction is executed to calculate the maximum utility of the subtree and $U 1$ is obtained (Step 8). In Step $9, U_{m_{\alpha}}^{M A X}$ records the maximum value of the subtree utility of $m_{\alpha}$ in the first for loop last time, and $U_{i}$ is the utility value obtained after the antennas are preallocated by the $B S_{i}$. The difference between the three is the utility improvement (Step 9) obtained after reconnection. Subsequently, the main algorithm judges whether the utility increment is greater than the previous maximum value; if so, it is replaced (Step 11) and $m$ is marked as a reconnected BS (Step 12). After all the loops of the second for loop are ended, if $i^{*}$ is obtained, the $B S_{i}$ is reconnected; $S E T_{i^{*}}$ and $U_{m_{\alpha}}^{M A X}$ are updated for the next comparison (Step 15).

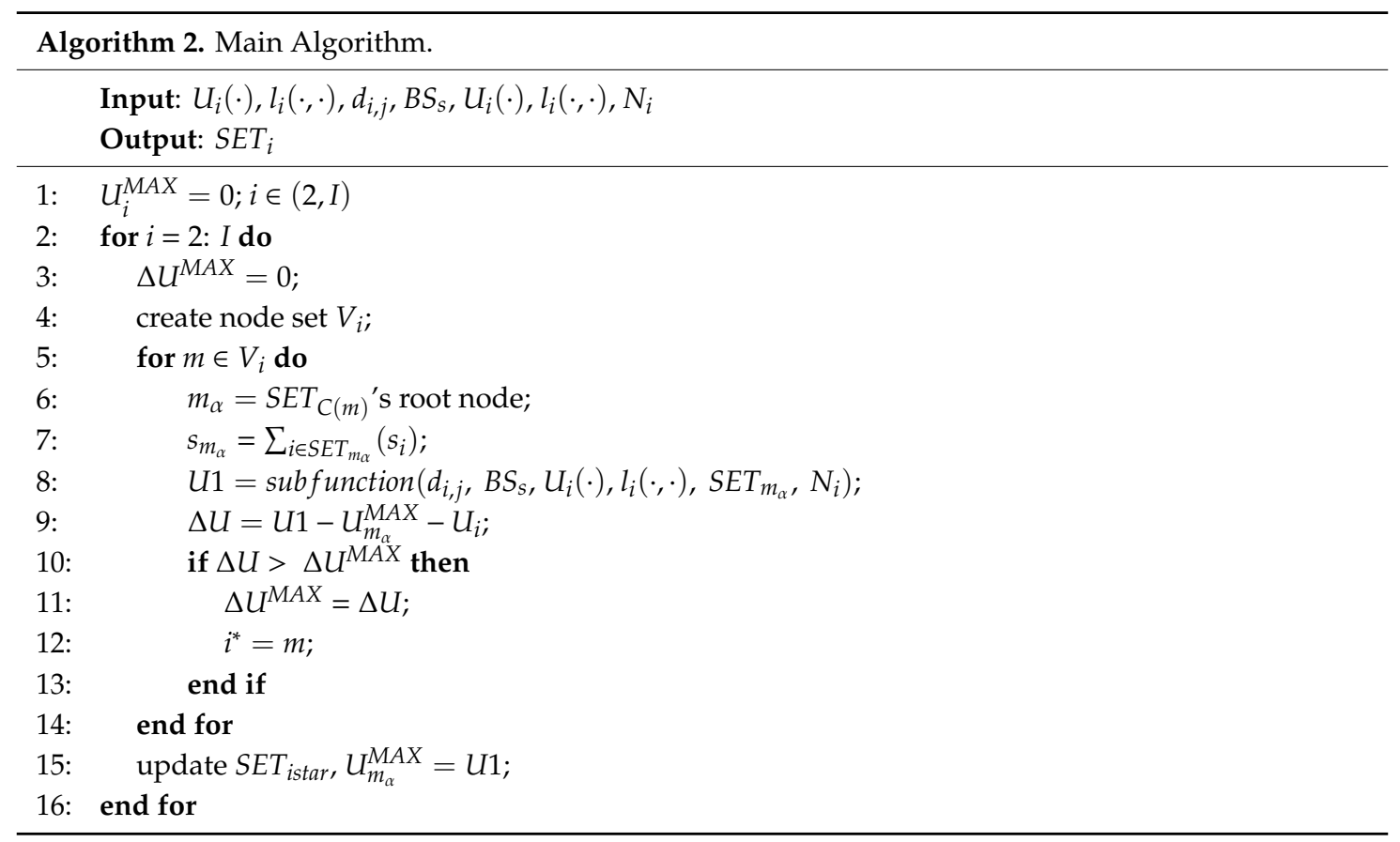

Finally, PSR calculates intermediate variable $U 1$, the maximum utility after $B S_{i}$ connected to BS of $V_{i}$ in subfunction in Algorithm 3. To find the total utility of the subtree, that is, the first two for loops (Step 2-20 and Step 3-19), all BSs allocate their $N_{i}$ antennas in the order from the closest to the farthest distance from the MBS. For the $n$-th antenna of $B S_{i}$, PSR sets a judgment factor $c$ (Step 4). When $c=0$, the number of antennas is not increased by the child node. Otherwise, one antenna is added when $c=1$. The value of $c$ is determined by the third for loop (Step 5-13). Then, the subfunction temporarily assigns the antenna to the child node $B S_{j}$ and obtains $A_{j}$ (Step 6). It checks if $r_{n}^{\Sigma}>\sum_{j \in \hat{i}} s_{j}$ $\& U\left(A_{j}\right)>U_{M A X}$ to ensure that the speed assigned to the child nodes does not exceed the uplink data rate (Step 7). Then, if the utility of the new allocation is greater than the known ones $U_{M A X}$, it replaces the old value (Step 8) and becomes the allocation candidate (Step 9-10). At this time, $j$ and $A_{j}$ are temporarily stored in $j^{*}$ and $A^{*}$, and $c$ is set to 1 (Step 11). After the third for loop, if $c=0$, the antenna allocation is unchanged (Step 14-15) because no better utility is obtained. However, if $c=1$, 
the antenna is allocated to $j^{*}$ (Step 17). Finally, $U 1$ is returned (Step 21). The signaling overhead of PSR is acceptable because of two reasons. First, the algorithm requires limited information. Each BS's candidate parents $V_{i}$ are only limited in an area. Second, the allocation results (each link's antenna number and data rate) of each BS do not take much overhead to transmit.

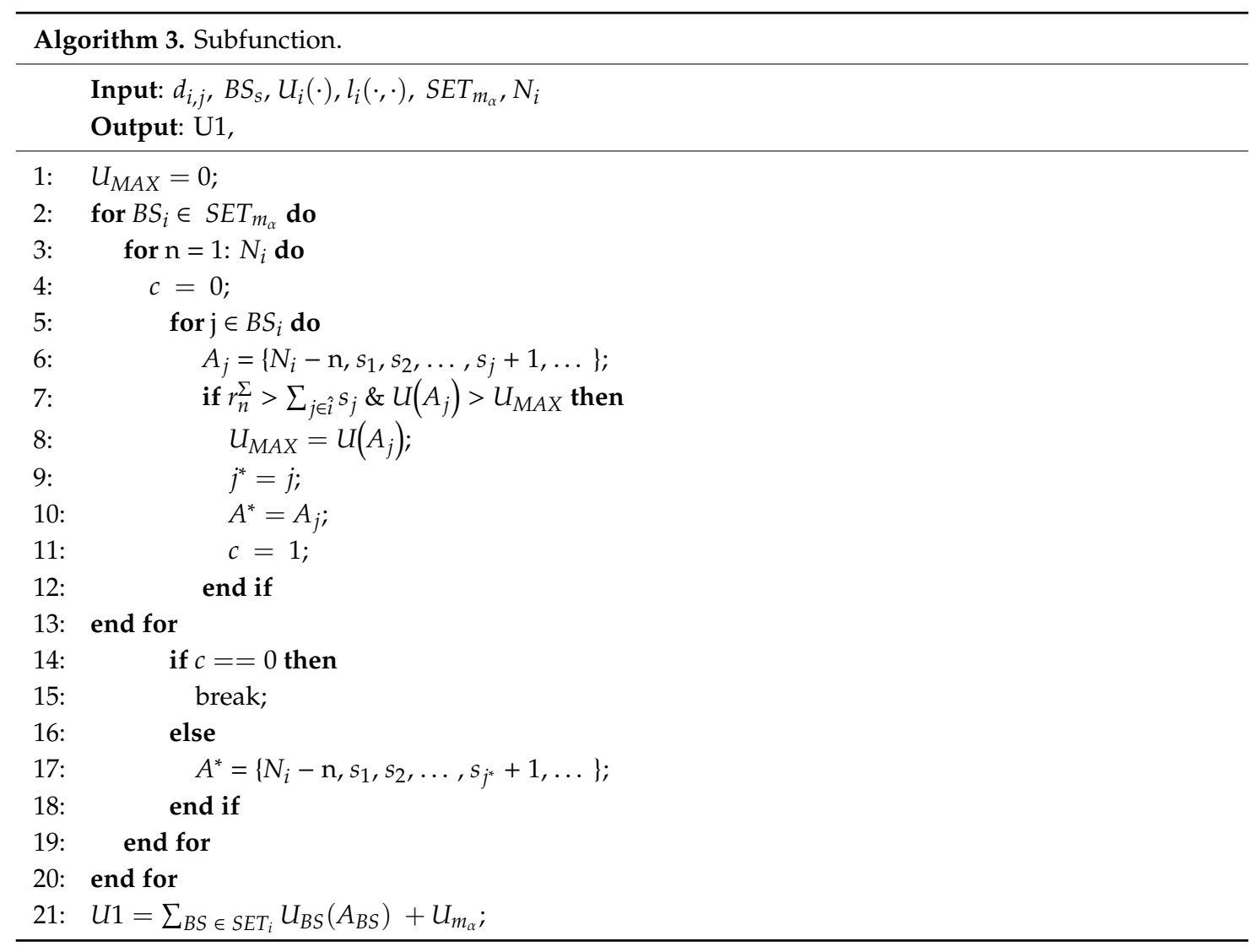

We again use Figure 3 to illustrate PSR operation. For simplicity of presentation, each BS is assumed to have only six antennas. In Algorithm 1 initialization, each BS connects to MBS and one antenna is achieved, i.e., $s_{2}=s_{3}=s_{4}=s_{5}=s_{6}=s_{7}=1$, as shown in Figure 3a. Because there is no $B S$ closer to $B S_{1}, B S_{2}$ connects to $B S_{1}$ directly. As the link between $B S_{2}$ and $B S_{3}$ requires more antennas than the link between $B S_{1}$ and $B S_{3}, B S_{2}$ cannot possibly become the candidate forwarder of $B S_{3}$. Therefore, there is a set of alternative nodes of $B S_{3}$, i.e., $V_{3}=\{\}$. As for $B S_{4}$, its candidate set is $V_{4}=\left\{B S_{2}, B S_{3}\right\}$. As shown by the white arrow in Figure $3 \mathrm{~b}$, PSR first connects $B S_{2}$ and adds $s_{4}$ to $s_{2}, s_{4}=0, s_{2}=2$, which leads to $A_{2}=\{5,2\}, A_{4}=\{6,1\}$, so the performance gain of this topology is $\Delta U=l_{2,1}(5,2)+l_{4,2}(6,1)-l_{2,1}(6,1)-l_{4,1}(6,1)$. Similarly, the algorithm calculates the difference when $B S_{4}$ connects to $B S_{3}$ for comparison. Assuming that connecting to $B S_{2}$ yields higher utility, the parent node of $B S_{4}$ is finally set as $B S_{2}$. The parent nodes of $B S_{5}, B S_{6}$, and $B S_{7}$ are also selected in the same way. As shown in Figure $3 c, B S_{5}$ is determined to connect to $B S_{3}$. $B S_{6}$ has candidate set of parent nodes $V_{6}=\left\{B S_{2}, B S_{4}\right\}$ and we assume that $B S_{2}$ is also a better choice, thus PSR determines the parent node of $B S_{6}$ as $B S_{2}$. Finally, as shown in Figure $3 \mathrm{~d}, B S_{7}$ is connected to $B S_{4}$, and the connection of the entire tree topology is completed. 


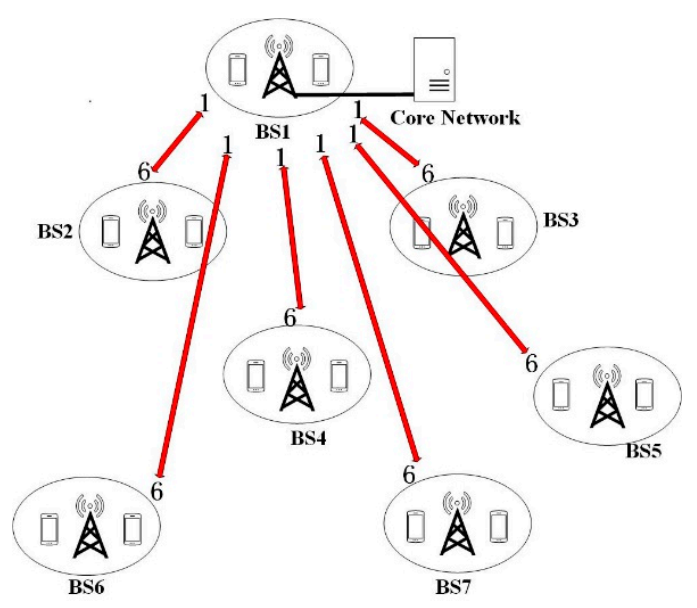

(a)

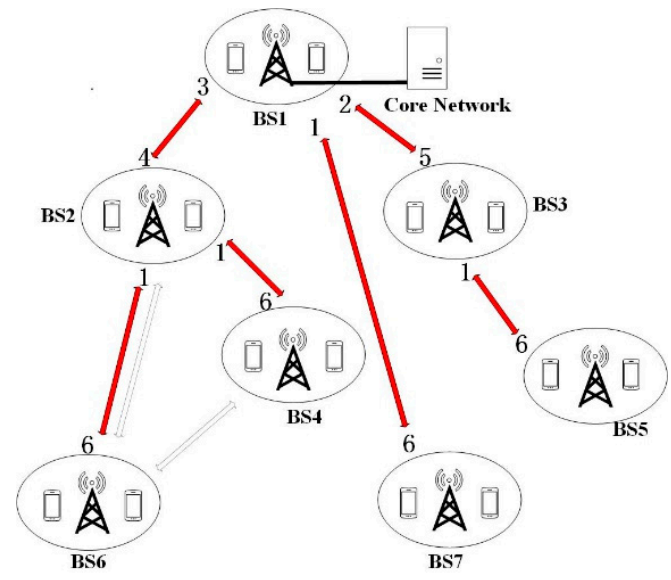

(c)

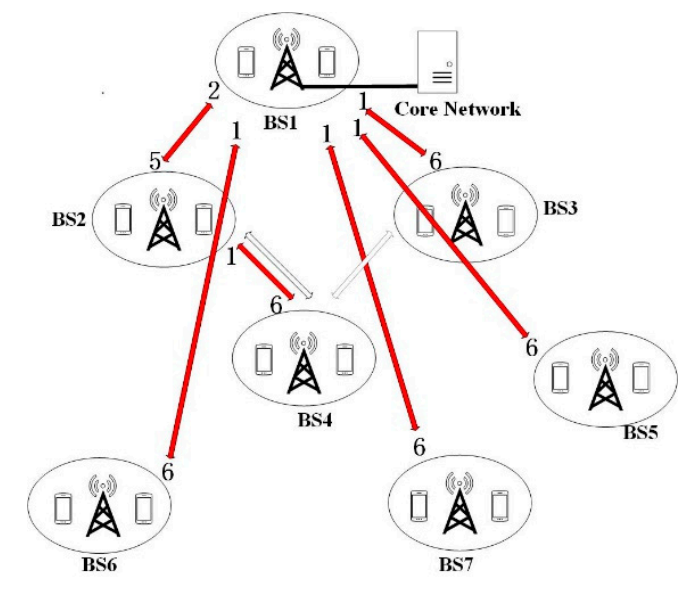

(b)

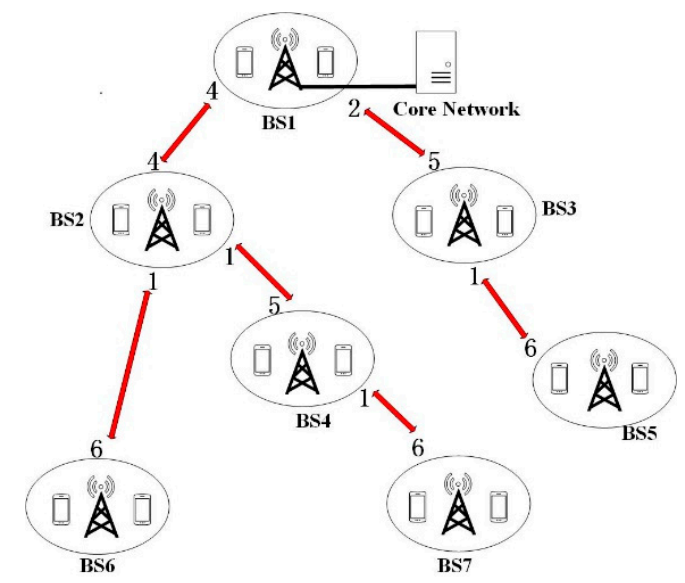

(d)

Figure 3. Example of PSR: (a) Initialization, (b) select parent node of $B S_{4}$, (c) select parent node of $B S_{5}$, (d) complete the entire topology.

\section{Numerical Results}

\subsection{Simulation Environment Settings}

In this section, we evaluate PSR's performance by simulation. As shown in Figure 4 , a $6 \times 6 \mathrm{~km}$ square area was generated. MBS $\left(B S_{1}\right)$ was placed in the center and other BSs were randomly distributed in the area. In order to avoid unrealistic approximation between BSs, the minimal distance between any two BSs was set as $0.3 \mathrm{~km}$. To observe the performance in difference distances, the overall simulation area was divided into three ranges: a circle with a diameter of $1 \mathrm{~km}$ (blue circle) was regarded as Area 1, nodes between the blue circle and the red circle (a circle with a diameter of $2 \mathrm{~km}$ ) were regarded as Area 2, and the residual area was regarded as Area 3.

The number of UE in each BS is set to 60. All other parameters are shown in Table 1. These parameters are very common and have been used in many other works with a similar environment [23]. In order to eliminate the possible adverse results caused by random factors, each setting was repeated 100 times, and the average value was acquired. The simulation software we used was MATLAB R2018a. Under the operating system Windows 10 and system setting of CPU AMD Ryzen5 2600U, 8G RAM, $I=60$ and $N_{i}=1200$, it took the PSR $12.6 \mathrm{~s}$ to execute the task 100 times. In other words, each single calculation was about 0.1-0.2 s, making PSR feasible for real-time and repeated execution to reflect the time-changing requirement caused by the mobility of UE. Table 1 summarizes the simulation parameters. 


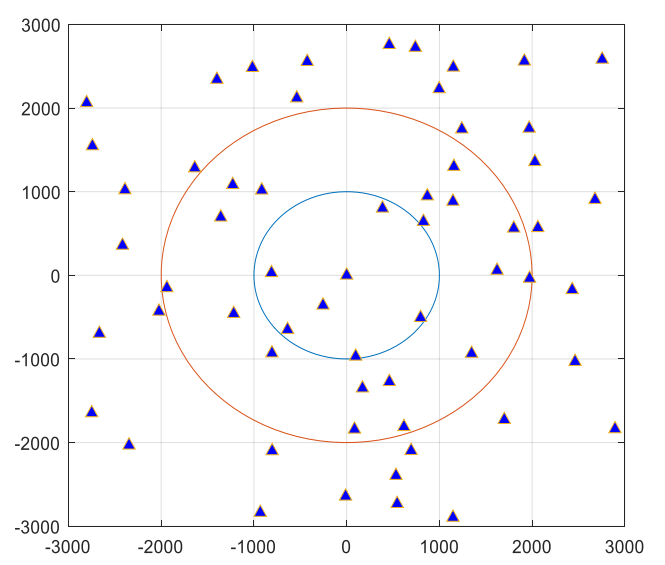

Figure 4. Simulation environment.

Table 1. Simulation parameters.

\begin{tabular}{cc}
\hline Parameter & Value \\
\hline Bandwidth (B) & $1000 \mathrm{MHz}$ \\
Transmit power (P) & $10 \mathrm{~W}$ \\
Noise density (D) & $-204 \mathrm{dBW} / \mathrm{Hz}$ \\
$l_{i}(s, t)$ & $\min (s, t) \cdot B_{A} \cdot \log _{2}\left(1+\frac{P_{A} \cdot 10^{-0.1 L_{i, j}}}{B_{A} \cdot 10^{0.1 D}}\right) \mathrm{bps}$ \\
Minimal distance between BSs & $250 \mathrm{~m}$ \\
The number of UE for each BS & 60 \\
\hline
\end{tabular}

Because there are no existing methods that dynamically combine antenna allocation and routing to provide an integrated solution, PSR was compared with two existing common routing schemes that we have discussed in the related work section: shortest path tree (SPT) and closest forward connection (CFC). SPT forms a shortest-path tree starting from the MBS, while each BS in CFC selects the shortest BS among the BSs that is more closer to the parent until it connects to the MBS. However, as these two schemes only establish topology and do not allocate antennas to form a complete solution, an antenna-allocation scheme Hierarchical Distributed Adjustment (HDA) [25] was used based on the topology they had generated. As shown in the analysis section as well as the simulation in [25], HDA is very efficient and provides a suboptimal solution that is very close to the optimum. Therefore, the comparison is fair and can show the significance of the joint consideration of topology and antenna allocation. Each BS's antennas were adjusted from 400 to 2000. As shown in [27], when the massive MIMO system uses regularized zero-forcing (RZF), point-to-point communication between two BSs with 400 antennas each can reach up to $82 \%$ of the system capacity limit. Although this may satisfy a point-to-point transmission, in the UWBR problem, where each BS has to connect to multiple BSs to form a tree topology, more antennas is necessary. Therefore, 400 antennas per BS was considered to be the lower bound in the simulation that our simulation started with.

\subsection{Simulation 1: Available Antennas}

In the first simulation, we observed the performance of the three methods under different antenna resources. The total BS, i.e., I, was set to 60 , while $N_{i}$ of each BS was adjusted from 400 to 2000 . The average utility and transmission speed (Mbps) of each area of each method are shown in Figure $5 \mathrm{a}, \mathrm{b}$. As $N_{i}$ increased, the average transmission speeds of all areas grew. Among them, BSs in the first area had obviously higher speeds in SPT and CFC because inner-layer BSs require fewer links and thus are considered more efficient to serve in SPT and CFC. On the other hand, PSR aims to maximize concave utility functions of each BS and serves BS in a more balanced way. When resources are scarce, it favors the BS with better channel quality. However, when it is served with certain amounts of resource and tends to saturate, it turns to those having more links or poorer channels. Thus, BSs in different layers 
had relatively approximate speed in PSR, and those in the third area had data rates higher than the other two schemes.

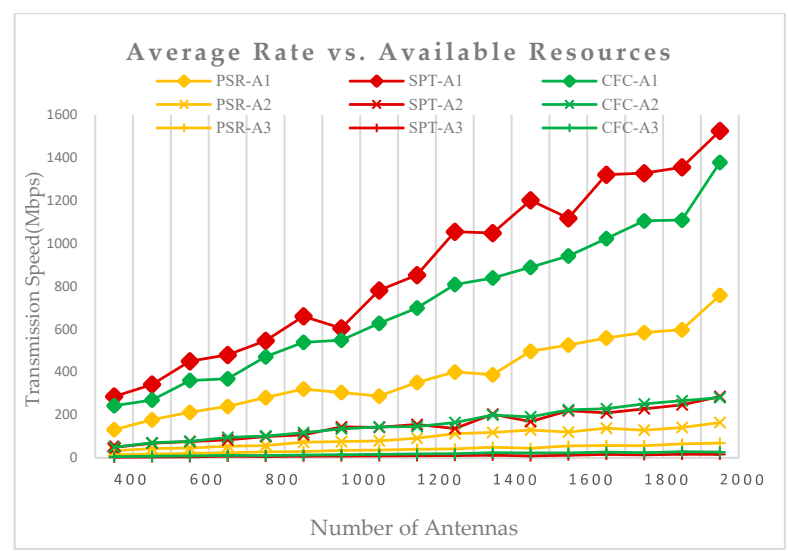

(a)

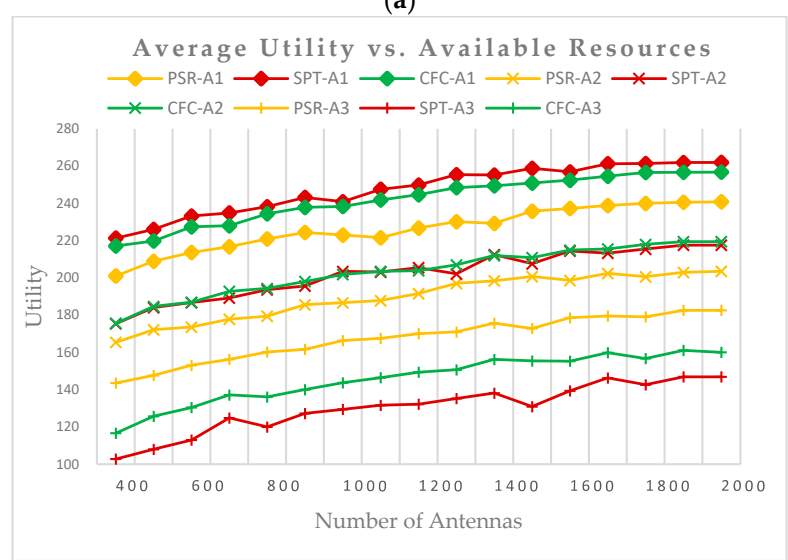

(b)

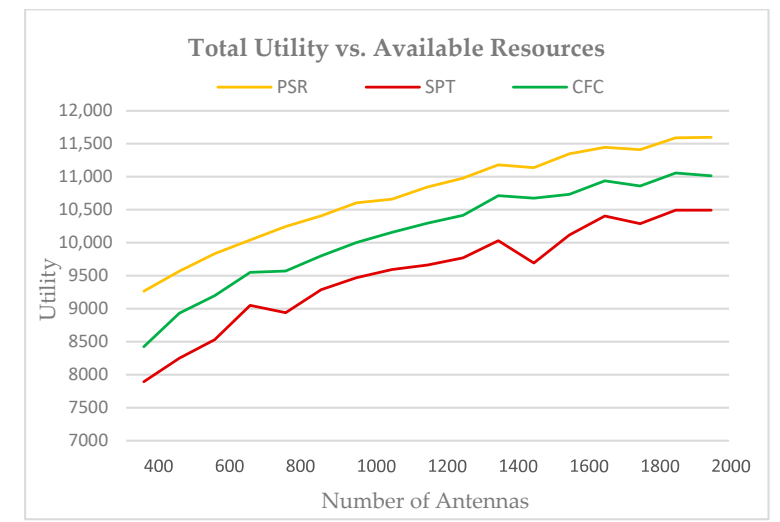

(c)

Figure 5. Performance of each area under different antennas: (a) data rate, (b) utility, (c) total utility.

Figure $5 \mathrm{c}$ shows the total utility of the system. The total utility of all methods was improved as the available resources increased. Among them, the total utility of PSR was significantly higher than the other two schemes because PSR is utility-oriented. The total utility of CFC was higher than SPT because there are many unnecessary short links in SPT that always find the shortest path of nodes.

\subsection{Simulation 2: Number of BSs}

Next, with the number of antennas fixed, we compared the transmission speed and the utility of the three methods under different populations. $N_{i}$ was fixed to 1200 , while I was adjusted from 50 to 80 . 
Figure 6a,b gives the transmission speed (Mbps) and average utility of each area. As I increased, there was no significant change in the utility and speed of the first and second areas of all three methods. This is because regardless of the routing schemes, a topology has to be built starting from the inner area; thus, the performance of inner BSs are not greatly affected by the increase of those in the outer areas. The average data rate and utility of the third area of PSR was roughly the same, while the average data rate and utility of SPT and CFC were significantly reduced. This is because SPT and CFC always favor inner areas. Thus, the speed of the outer areas is sacrificed when BSs of the first and second area increases. In contrast, as PSR focuses on the overall utility benefit, it can adjust antennas more efficiently and provides a milder performance drop of the outer BSs.

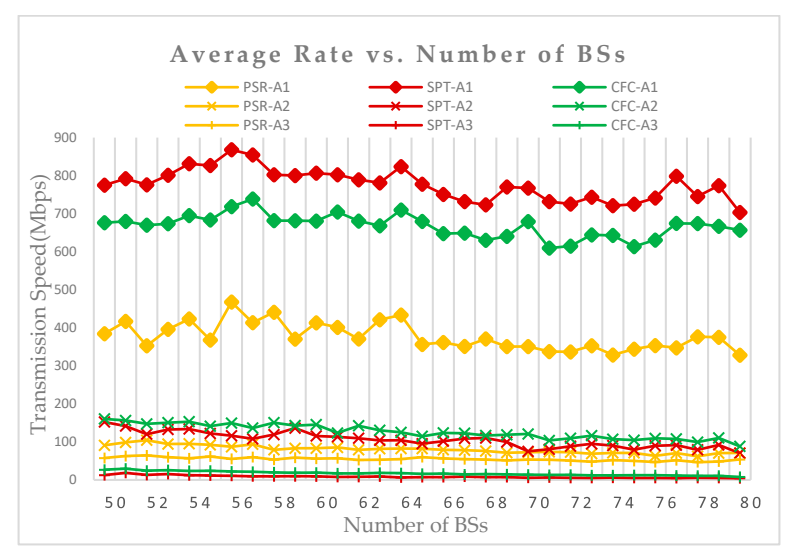

(a)

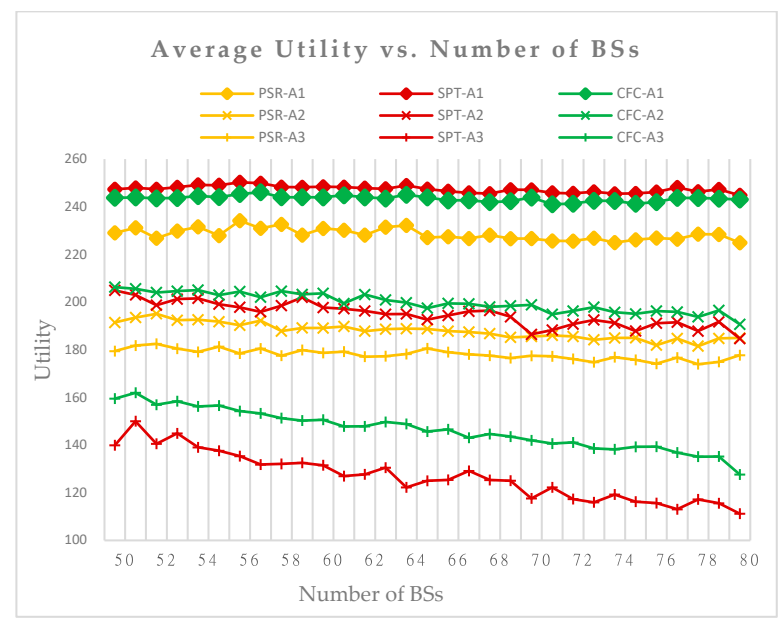

(b)

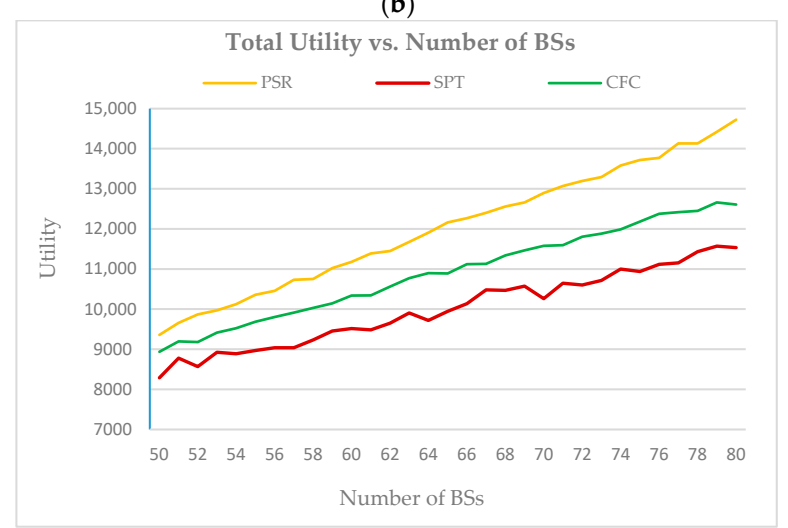

(c)

Figure 6. Performance of each area under different number of base stations (BSs): (a) data rate, (b) utility, (c) total utility. 
Figure $6 \mathrm{c}$ shows that the total utility of all three methods was improved with the increase in the number of BSs. Although the average speed would be reduced with the increase of BSs, the average marginal utility of BSs increased as more BSs require more resources. However, the utility of SPT was the lowest because its links were too short. PSR again provided the highest total utility due to its flexibility of allocation. The results also showed that a higher total/average data rate did not always lead to better system utility because the resources also needed to be allocated fairly to prevent monopolization and starvation.

\subsection{Simulation 3: Performance Comparison}

Finally, we examined the speed distribution of BSs of the three methods. Due to the fixed number of UE, only the data rate of each BS was compared. We set $I$ to 60 and $N_{i}$ to 1200 . Figure 7 shows the cumulative distribution function (CDF) of the data rate of the three methods. To quantify the fairness of the performance distribution, a well-known fairness metric called Jain's index was used here. Given a set of numbers $\left[x_{1}, x_{2}, \ldots, x_{n}\right]$, Jain's index $\frac{\left(\sum_{i=1}^{n} x_{i}\right)^{2}}{n \cdot \sum_{i=1}^{n}\left(x_{i}^{2}\right)}$ ranges from $\frac{1}{n}$ (worst case) to 1 (best case). When each member of the set has equal value, the index becomes one. As shown in Table 2, PSR had the highest Jain's index. About $80 \%$ of the three methods were concentrated in a range of $0-100 \mathrm{Mbps}$. The third area was large and had many low-speed BSs. Although SPT and CFC had very high inner BSs, their outer BSs had extremely low data rate, and about $60 \%$ were concentrated below $20 \mathrm{Mbps}$. This caused many BSs to work in a low-utility condition, making the overall system less effective. Due to PSR's utility-oriented behavior, it provided a higher outer data rate, concentrated near $50 \mathrm{Mbps}$. Because of the strong marginal effect of the utility, even when the speed was doubled at low speed, it could get several times more utility improvement.

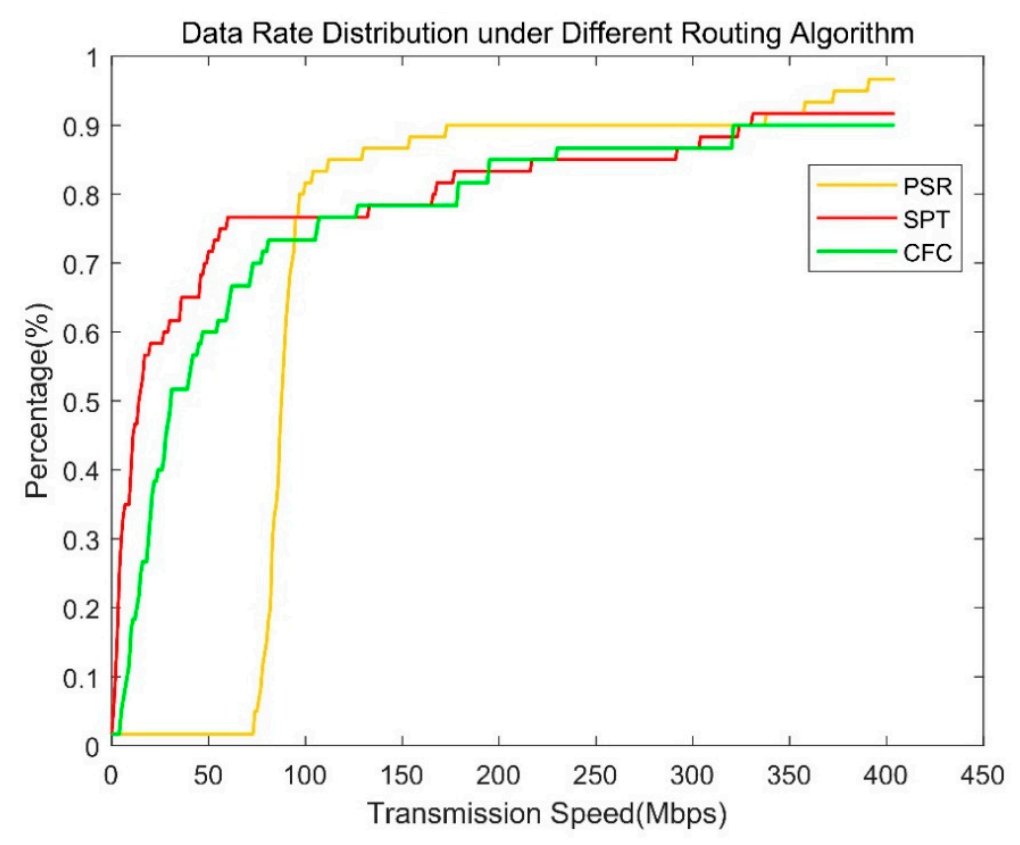

Figure 7. Distribution of different methods.

Table 2. Value of Jain's index for each mechanism.

\begin{tabular}{cccc}
\hline & PSR & SPT & CFC \\
\hline Jain's index & 0.5243 & 0.1499 & 0.2107 \\
\hline
\end{tabular}




\section{Conclusions and Future Work}

In this paper, we studied a brand-new issue: the joint consideration of route establishment and antenna allocation in heterogeneous massive MIMO backhaul networks. Given the number of UE of each BS, the available antennas, and the distance between all BSs, we introduced the new problem, i.e., utility-based wireless backhaul routing (UWBR), which involves adjusting the antennas of each link and selecting routes to improve overall user utility with antennas, links, and data-rate constraints. To tackle this problem, we proposed a heuristic algorithm called PSR. In this algorithm, all BSs are connected to the MBS, and antennas of the MBS are preallocated to the BSs. Then, each BS selects its candidate parents that yield the largest utility difference if reconnected to its subtree. Using PSR, the overall utility is improved, and the outer BSs can obtain better data rate and utility performance. We proved that UWBR is an NPC problem and analyzed the maximum utility that the system can possibly achieve. The simulation results show that our algorithm is superior to other existing schemes in terms of the data rate and total utility of the outer BSs. As the channel is modeled in a general way, this algorithm can be applied to different types of massive MIMO wireless mesh networks.

There are still some issues to be discussed in the future. First, links with different uplink and downlink requirements seem to be an interesting issue that is worth considering. In addition, this work can be extended by considering the detail signaling overhead and the mobility of user equipment and base stations.

Author Contributions: Conceptualization, W.-H.K. and W.Z.; methodology, W.-H.K. and W.Z.; software, W.Z.; validation, W.-H.K.; formal analysis, W.Z.; writing—original draft preparation, W.Z.; writing—review and editing, W.-H.K. and W.Z. All authors have read and agreed to the published version of the manuscript.

Funding: This research was funded by Ministry of Science and Technology, grant number 107-2221-E-155-024-MY3.

Conflicts of Interest: The authors declare no conflict of interest.

\section{Appendix A}

The problem introduced in [23], i.e., utility-based wireless backhaul routing (UBAA), is an NP-hard problem. Its network topology is fixed, and only the antennas need to be allocated. However, UWBR needs to adjust the topology on the basis of UBAA. After adjusting the topology, the antennas need to be redistributed. Therefore, UWBR is more complicated than UBAA, so UWBR is also NP-hard.

We considered the following situation: one MBS $B S_{1}$ and two common $B S_{2}$ and $B S_{3}$; each BS has two antennas. Here, the routing determination and antenna allocation needs the following steps. Firstly, both $B S_{2}$ and $B S_{3}$ are preallocated to one antenna. Secondly, $B S_{2}$ connects to $B S_{1}$, and $B S_{3}$ can choose to connect to $B S_{1}$ or $B S_{2}$. Therefore, there are only two cases: $U_{1}^{M A X}=U_{2}\left(l_{2,1}(1,2)\right)+U_{3}\left(l_{3,1}(1,2)\right)$ or $U_{1}^{M A X}=U_{2}\left(l_{2,1}(2,1)\right)+U_{3}\left(l_{3,2}(1,2)\right)$; the complexity of the whole step is $\mathrm{O}(|i|+|i|)=\mathrm{O}(|i|)$, which is in polynomial time. Because UWBR is both NP-hard and NP, it is NP-complete.

\section{Appendix B}

The highest possible data rate at which the MBS connects to the core network through the wired network is $l_{2,1}\left(N_{i}, N_{i}\right)$. Assuming that all non-MBSs can get the data rate of $\frac{l_{2,1}\left(N_{i}, N_{i}\right)}{I-1}$, the highest system utility $(I-1) \cdot U\left(\frac{\left(l_{2,1}\left(N_{i}, N_{i}\right)\right.}{I-1}\right)$ can be obtained. Because the utility function is concave, that is, $\forall r$ :

$$
U(r+3)-U(r+2)<U(r+2)+U(r+1)
$$

when the speed between BSs can be guaranteed, if there is $r_{i}>\frac{l_{2,1}\left(N_{i}, N_{i}\right)}{I-1}$, there must be $r_{j}<\frac{l_{2,1}\left(N_{i}, N_{i}\right)}{I-1}$, then

$$
U\left(r_{i}\right)-U\left(\frac{l_{2,1}\left(N_{i}, N_{i}\right)}{I-1}\right)<U\left(\frac{l_{2,1}\left(N_{i}, N_{i}\right)}{I-1}\right)+U\left(r_{j}\right) .
$$


Therefore, when the speed of all BSs reaches $\frac{l_{2,1}\left(N_{i}, N_{i}\right)}{I-1}$, the maximized utility of the entire system is $(I-1) \cdot U\left(\frac{l_{2,1}\left(N_{i}, N_{i}\right)}{I-1}\right)$. However, due to the number of antennas and distance between BS limitations, it is impossible to reach the maximum, then

$$
U_{M A X}<(I-1) \cdot U\left(\frac{l_{2,1}\left(N_{i}, N_{i}\right)}{I-1}\right)
$$

\section{References}

1. Andrews, J.G.; Buzzi, S.; Choi, W.; Hanly, S.V.; Lozano, A.; Soong, A.C.; Zhang, J.C. What Will 5G Be? IEEE J. Sel. Areas Commun. 2014, 32, 1065-1082. [CrossRef]

2. Zhang, Q.; Jin, S.; McKay, M.; Morales-Jimenez, D.; Zhu, H. Power Allocation Schemes for Multicell Massive MIMO Systems. IEEE Trans. Wirel. Commun. 2015, 14, 5941-5955. [CrossRef]

3. Zhao, J.; Quek, T.Q.S.; Lei, Z. Heterogeneous Cellular Networks Using Wireless Backhaul: Fast Admission Control and Large System Analysis. IEEE J. Sel. Areas Commun. 2015, 33, 2128-2143. [CrossRef]

4. Tabassum, H.; Sakr, A.H.; Hossain, E. Analysis of Massive MIMO-Enabled Downlink Wireless Backhauling for Full-Duplex Small Cells. IEEE Trans. Commun. 2016, 64, 2354-2369. [CrossRef]

5. Beyranvand, H.; Lim, W.; Maier, M.; Verikoukis, C.; Salehi, J.A. Backhaul-Aware User Association in FiWi Enhanced LTE-A Heterogeneous Networks. IEEE Trans. Wirel. Commun. 2015, 14, 2992-3003. [CrossRef]

6. Chen, D.C.; Quek, T.Q.S.; Kountouris, M. Backhauling in Heterogeneous Cellular Networks: Modeling and Tradeoffs. IEEE Trans. Wirel. Commun. 2015, 14, 3194-3206. [CrossRef]

7. Hong, W.; Tsai, Z. A Multichannel Scheduler for High-Speed Wireless Backhaul Links with Packet Concatenation. IEEE Trans. Mob. Comput. 2010, 9, 201-214. [CrossRef]

8. Yang, F.; Luo, X. A Restless MAB-Based Index Policy for UL Pilot Allocation in Massive MIMO over Gauss-Markov Fading Channels. IEEE Trans. Veh. Technol. 2020, 69, 3034-3047. [CrossRef]

9. Mosleh, S.; Almosa, H.; Perrins, E.; Liu, L. Downlink Resource Allocation in Cell-Free Massive MIMO Systems. In Proceedings of the 2019 International Conference on Computing, Networking and Communications (ICNC), Honolulu, HI, USA, 11 April 2019; pp. 883-887. [CrossRef]

10. Nguyen, T.M.; Ha, V.N.; Le, L.B. Resource Allocation Optimization in Multi-User Multi-Cell Massive MIMO Networks Considering Pilot Contamination. IEEE Access 2015, 3, 1272-1287. [CrossRef]

11. Buzzi, S.; D'Andrea, C.; Zappone, A.; D'Elia, C. User-Centric 5G Cellular Networks: Resource Allocation and Comparison with the Cell-Free Massive MIMO Approach. IEEE Trans. Wirel. Commun. 2020, 19, 1250-1264. [CrossRef]

12. Chen, Z.; Björnson, E.; Larsson, E.G. Dynamic Resource Allocation in Co-Located and Cell-Free Massive MIMO. IEEE Trans. Green Commun. Netw. 2020, 4, 209-220. [CrossRef]

13. Ngo, H.Q.; Matthaiou, M.; Larsson, E.G. Massive MIMO with Optimal Power and Training Duration Allocation. IEEE Wirel. Commun. Lett. 2014, 3, 605-608. [CrossRef]

14. Huang, K.; Wang, Z.; Wan, X.; Fan, Z.; Xu, Y. Max-Min Resource Allocation for Wireless Power Transfer Enabled Massive MIMO Systems. In Proceedings of the 2019 IEEE 5th International Conference on Computer and Communications (ICCC), Chengdu, China, 13 April 2019; pp. 889-893. [CrossRef]

15. Khan, I.; Zafar, M.H.; Jan, M.T.; Lloret, J.; Basheri, M.; Singh, D. Spectral and Energy Efficient Low-Overhead Uplink and Downlink Channel Estimation for 5G Massive MIMO Systems. Entropy 2018, 20, 92. [CrossRef]

16. Saraereh, O.A.; Khan, I.; Alsafasfeh, Q.; Alemaishat, S.; Kim, S. Low-Complexity Channel Estimation in 5G Massive MIMO-OFDM Systems. Symmetry 2019, 11, 713. [CrossRef]

17. Ogawa, H.; Tran, G.K.; Sakaguchi, K.; Haustein, T. Traffic Adaptive Formation of mmWave Meshed Backhaul Networks. In Proceedings of the 2017 IEEE International Conference on Communications Workshops (ICC Workshops), Paris, UK, 3 July 2017; pp. 185-191. [CrossRef]

18. Li, Y.; Cai, A.; Qiao, G.; Shi, L.; Bose, S.K.; Shen, G. Multi-Objective Topology Planning for Microwave-Based Wireless Backhaul Networks. IEEE Access 2016, 4, 5742-5754. [CrossRef]

19. Kuo, F.; Zdarsky, F.A.; Lessmann, J.; Schmid, S. Cost-Efficient Wireless Mobile Backhaul Topologies: An Analytical Study. In Proceedings of the 2010 IEEE Global Telecommunications Conference GLOBECOM 2010, Miami, FL, USA, 10 January 2010; pp. 1-5. [CrossRef] 
20. Nasr, I.; Fahmy, Y. Millimeter-Wave Wireless Backhauling for 5G Small Cells: Scalability of Mesh over Star Topologies. In Proceedings of the 2017 IEEE 18th International Symposium on A World of Wireless, Mobile and Multimedia Networks (WoWMoM), Macau, China, 13 July 2017; pp. 1-6. [CrossRef]

21. Chen, D.; Schuler, J.; Wainio, P.; Salmelin, J. 5G Self-optimizing Wireless Mesh backhaul. In Proceedings of the 2015 IEEE Conference on Computer Communications Workshops (INFOCOM WKSHPS), Hong Kong, China, 6 August 2015; pp. 23-24. [CrossRef]

22. Scopelliti, P.; Tropeano, A.; Muntean, G.; Araniti, G. An Energy-quality utility-based adaptive scheduling solution for mobile users in dense networks. IEEE Trans. Broadcast. 2020, 66, 47-55. [CrossRef]

23. Pham, Q.; Hwang, W. Network Utility Maximization-Based Congestion Control over Wireless Networks: A Survey and Potential Directives. IEEE Commun. Surv. Tutor. 2017, 19, 1173-1200. [CrossRef]

24. Tan, L.; Zhu, Z.; Ge, F.; Xiong, N. Utility Maximization Resource Allocation in Wireless Networks: Methods and Algorithms. IEEE Trans. Syst Man Cybern. Syst. 2015, 45, 1018-1034. [CrossRef]

25. Kuo, W.; Chen, C. Distributed Antenna Allocation Scheme for Massive MIMO Cellular Backhaul Networks. IEEE Access 2018, 6, 73895-73904. [CrossRef]

26. Evolved Universal Terrestrial Radio Access (E-UTRA); Mobility Enhancements in Heterogeneous Networks, Release 11, document TR 36.839 V11.1.0, 3GPP, 2012. 3GPP. Available online: https://www.3gpp.org/ specifications/releases/69-release-11 (accessed on 16 October 2020).

27. Hoydis, J.; Ten Brink, S.; Debbah, M. Massive MIMO in the UL/DL of cellular networks: How many Antennas do we need? IEEE J. Sel. Areas Commun. 2013, 31, 160-171. [CrossRef]

Publisher's Note: MDPI stays neutral with regard to jurisdictional claims in published maps and institutional affiliations.

(C) 2020 by the authors. Licensee MDPI, Basel, Switzerland. This article is an open access article distributed under the terms and conditions of the Creative Commons Attribution (CC BY) license (http://creativecommons.org/licenses/by/4.0/). 